\title{
Marchenko imaging: Imaging with primaries, internal multiples, and free-surface multiples
}

\author{
Satyan Singh ${ }^{1}$, Roel Snieder ${ }^{1}$, Jyoti Behura ${ }^{2}$, Joost van der Neut ${ }^{3}$, \\ Kees Wapenaar ${ }^{3}$, and Evert Slob ${ }^{3}$
}

\begin{abstract}
Recent work on retrieving the Green's function with the Marchenko equation shows how these functions for a virtual source in the subsurface can be obtained from reflection data. The response to the virtual source is the Green's function from the location of the virtual source to the surface. The Green's function is retrieved using only the reflection response of the medium and an estimate of the first arrival at the surface from the virtual source. Current techniques, however, only include primaries and internal multiples. Therefore, all surface-related multiples must be removed from the reflection response prior to Green's function retrieval. We have extended the Marchenko equation to retrieve the Green's function that includes primaries, internal multiples, and free-surface multiples. In other words, we have retrieved the Green's function in the presence of a free surface. The information needed for the retrieval is the same as the current techniques, with the only difference being that the reflection response now also includes free-surface multiples. The inclusion of these multiples makes it possible to include them in the imaging operator, and it obviates the need for surfacerelated multiple elimination. This type of imaging with Green's functions is called Marchenko imaging.
\end{abstract}

\section{INTRODUCTION}

To focus a wavefield at a point in a medium only requires surface reflection data and an estimate of the first-arriving wave at the sur- face from a point source at the focusing location (Broggini et al., 2012; Broggini and Snieder, 2012; Wapenaar et al., 2013a). Unlike in seismic interferometry (Bakulin and Calvert, 2006; Wapenaar and Fokkema, 2006), no receiver is required at the desired focusing location, i.e., the virtual source location and single-sided illumination suffices. Significantly, the detailed medium parameters need not be known to focus the wavefield as opposed to the time-reversal method, which requires the knowledge of detailed medium parameters and enclosing boundaries for correct focusing. However, the traveltime of the direct arrival of the virtual source to the surface is required for Green's function retrieval. To obtain an estimate of this traveltime, one only needs a macromodel of the velocity.

The focusing scheme of Broggini et al. (2012), Broggini and Snieder (2012), and Wapenaar et al. (2013a) is an extension of the algorithm of Rose (2002a, 2002b), who shows an iterative scheme that solves the Marchenko equation for wavefield focusing in 1D. The focused events in the wavefield for the virtual source consist of primaries and internal multiples (Wapenaar et al., 2013a) but not free-surface multiples. Importantly, Rose (2002a, $2002 b$ ) derives the focusing method for single-sided illumination with sources and receivers on one side of the medium, similar to current geophysical acquisition methods.

Wapenaar et al. (2011) illustrate imaging with the Green's function in 1D and also discuss how to image in multidimensions (2D and 3D). Similarly, Behura et al. (2012) introduce a correlation-imaging algorithm based on the Green's function retrieval scheme that images not only primaries but also internal multiples. Broggini et al. (2014) extend the work of Behura et al. (2012) by using multidimensional deconvolution (MDD) as the imaging condition in place of conventional crosscorrelation or deconvolution, which further reduces the artifacts. Central in these methods is the retrieval of the Green's function from the acquisition surface to any point in the

Manuscript received by the Editor 16 October 2014; revised manuscript received 11 April 2015; published online 17 July 2015.

${ }^{1}$ Colorado School of Mines, Center for Wave Phenomena, Department of Geophysics, Golden, Colorado, USA. E-mail: sasingh@mines.edu; rsnieder@ mines.edu.

${ }^{2}$ Formerly Colorado School of Mines, Center for Wave Phenomena, Department of Geophysics, Golden, Colorado, USA; presently Seismic Science LLC, Colorado, USA. E-mail: jyotibehura@ gmail.com.

${ }^{3}$ Delft University of Technology, Department of Geoscience and Engineering, Delft, The Netherlands. E-mail: j.r.vanderneut@tudelft.nl; c.p.a.wapenaar@ tudelft.nl; e.c.slob@tudelft.nl.

(C) 2015 Society of Exploration Geophysicists. All rights reserved. 
medium. This Green's function is essentially a redatuming or downward continuation operator. Because this Green's function includes primaries and internal multiples, we expect improved subsurface images compared with using primaries alone.

We summarize our work in Figure 1. In this paper, any variable with a subscript zero (e.g., $R_{0}$ ) indicates that no free surface is present. As shown in Figure 1, prior to the algorithm of Broggini et al. (2012), one must remove the free-surface multiples from the reflection response of the medium (solid upgoing arrow) to retrieve the Green's function. The removal of the free-surface multiples can be achieved by surface-related multiple elimination (SRME) (Verschuur et al., 1992). In our study, we modify the earlier focusing algorithms (Rose, 2002a; Broggini et al., 2012; Wapenaar et al., 2013a) to focus not only primaries and internal multiples but also the free-surface multiples; this is labeled "Our work" in Figure 1. We achieve such focusing using the reflection response $R$ in the presence of a free surface and an estimate of the first arrival from the focus location to the surface. Notably, our proposed Green's function retrieval scheme obviates the need for SRME (see Figure 1) to construct the Green's function.

The free surface is the strongest reflector in the system; therefore, in general, the free-surface multiples are stronger than internal multiples. In addition, free-surface multiples can be used to provide better illumination, higher fold, and better vertical resolution of the subsurface (Schuster et al., 2003; Jiang et al., 2007; Muijs et al., 2007a, 2007b). For these reasons, by retrieving the Green's function, which includes primaries and all multiples (including free-surface multiples), and using the imaging condition proposed by Behura et al. (2012) and by Broggini et al. (2014), we expect better imaging of the subsurface.

\section{THEORY}

In this section, we derive the main equations for the retrieval of the Green's function in the presence of a free surface, with the detailed derivations in Appendix A. We first summarize the existing theory that this paper is building on.

The theory of focusing the wavefield without a free surface, i.e., retrieving the Green's function $G_{0}$ from $R_{0}$, is covered by Rose

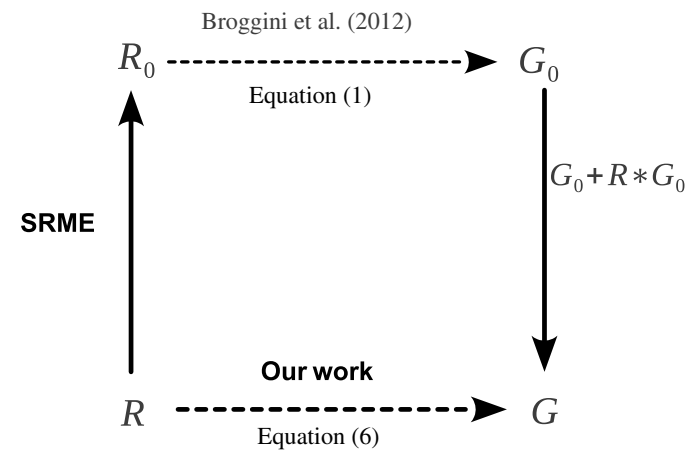

Figure 1. Overview of the methods to focus the wavefield using an iterative approach. The $R$ denotes reflected waves recorded at the surface in the presence of a free surface, and $R_{0}$ is the reflection response for a medium without a free surface. The $G$ is the Green's function at the surface for a virtual source located at a point in the medium in the presence of a free surface, and $G_{0}$ is the Green's function in the absence of a free surface. The two dashed arrows indicate separate iterative schemes. (2002a), Broggini et al. (2012), and Wapenaar et al. (2013a). As summarized in Figure 1, we have to remove the free-surface multiples from the reflection response $R$ (for instance, by SRME) to get $R_{0}$ and then compute $G_{0}$, the Green's function in the absence of the free surface.

Wapenaar et al. (2004) show that we can relate the transmission operators for media with and without the free surface. Similarly, we can retrieve $G$ (the Green's function in the presence of the free surface) from $G_{0}$ in the frequency domain with the expression

$$
\begin{aligned}
G\left(\mathbf{x}_{\mathbf{i}}^{\prime}, \mathbf{x}_{\mathbf{0}}, \omega\right)= & G_{0}\left(\mathbf{x}_{\mathbf{i}}^{\prime}, \mathbf{x}_{\mathbf{0}}, \omega\right) \\
& -\int_{\partial D_{0}} G_{0}\left(\mathbf{x}_{\mathbf{i}}^{\prime}, \mathbf{x}, \omega\right) R\left(\mathbf{x}, \mathbf{x}_{\mathbf{0}}, \omega\right) d \mathbf{x},
\end{aligned}
$$

where $\partial D_{0}$ is the acquisition surface, $\mathbf{x}_{\mathbf{0}}$ and $\mathbf{x}_{\mathbf{i}}^{\prime}$ are spatial positions along $\partial D_{0}$ and $\partial D_{i}$ (an arbitrary depth level, below $\partial D_{0}$ ), and $R$ is the reflection response for a downgoing incident wavefield at $\partial D_{0}$. The arbitrary depth levels are defined in the same way as in the work of Wapenaar et al. (2004). In our case, we replace the transmission responses (in equation 22 of Wapenaar et al., 2004) with the corresponding Green's functions $G$ or $G_{0}$ because the Green's function is the total transmitted wavefield from the focusing point to the surface. Note that this approach, $R \rightarrow R_{0} \rightarrow G_{0} \rightarrow G$, follows the tortuous path shown in Figure 1. We can, however, retrieve the Green's function in the presence of the free surface directly from the measured reflection data $R \rightarrow G$ (Figure 1, black dashed arrow). Therefore, $R \rightarrow G$ avoids SRME and the retrieval of $G_{0}$. We generalize the formulation of Wapenaar et al. (2013a) $\left(R_{0} \rightarrow G_{0}\right)$ to include free-surface multiples $(R \rightarrow G)$; the detailed mathematics of this retrieval is documented in Appendix A. The reflections from the free surface are included in the focusing scheme, similar to the treatment by Wapenaar et al. (2004) of free-surface multiples.

We define our spatial coordinates by their horizontal and depth components, for instance, $\mathbf{x}_{\mathbf{0}}=\left(\mathbf{x}_{\mathbf{H}}, x_{3,0}\right)$, where $\mathbf{x}_{\mathbf{H}}$ denotes the horizontal coordinates $\left(x_{1}, x_{2}\right)$ at a depth $x_{3,0}$. We define solutions of the wave equation that focus at a point in a medium, and we refer to these as the focusing functions $f_{1}$ and $f_{2}$. The $f_{1}$ function involves waves that focus at $\mathbf{x}_{\mathbf{i}}^{\prime}$ at a defined depth level $\left(\partial D_{i}\right)$ for incoming and outgoing waves at the acquisition surface $\left(\partial D_{0}\right)$ at $\mathbf{x}_{\mathbf{0}}$ (Figure 2a). The function $f_{2}$ is a solution for waves that focus just above $\partial D_{0}$ at $\mathbf{x}_{\mathbf{0}}^{\prime \prime}$ for incoming and outgoing waves at $\partial D_{i}$ (Figure 2b).

The focusing functions are auxiliary wavefields, which exist in a reference medium that has the same material properties as the actual inhomogeneous medium between $\partial D_{0}$ and $\partial D_{i}$ and that is homogeneous above $\partial D_{0}$ and reflection-free below $\partial D_{i}$ (Slob et al., 2014). Therefore, the boundary conditions on $\partial D_{0}$ and $\partial D_{i}$ in the reference medium, where the focusing function exists, are reflection free.

Note that this boundary condition need not be the same as the actual medium. The focusing functions can be separated into upand downgoing waves; the first focusing function in the frequency domain reads

$$
f_{1}\left(\mathbf{x}, \mathbf{x}_{\mathbf{i}}^{\prime}, \omega\right)=f_{1}^{+}\left(\mathbf{x}, \mathbf{x}_{\mathbf{i}}^{\prime}, \omega\right)+f_{1}^{-}\left(\mathbf{x}, \mathbf{x}_{\mathbf{i}}^{\prime}, \omega\right),
$$

whereas the second focusing function reads

$$
f_{2}\left(\mathbf{x}, \mathbf{x}_{\mathbf{0}}^{\prime \prime}, \omega\right)=f_{2}^{+}\left(\mathbf{x}, \mathbf{x}_{\mathbf{0}}^{\prime \prime}, \omega\right)+f_{2}^{-}\left(\mathbf{x}, \mathbf{x}_{\mathbf{0}}^{\prime \prime}, \omega\right) .
$$


In this paper, the superscript + refers to downgoing waves and refers to upgoing waves at the observation point $\mathbf{x}$.

Table 1 and Figure 2a show the one-way wavefields for the $f_{1}$ function at the depth levels $\partial D_{0}$ and $\partial D_{i}$, which we define in wave state A. We call these waves one way because locally these fields are strictly up- or downgoing at the observation point; however, their coda will include the up- and downgoing waves.

The focusing function $f_{1}^{+}\left(\mathbf{x}, \mathbf{x}_{\mathbf{i}}^{\prime}, t\right)$ is shaped such that $f_{1}\left(\mathbf{x}, \mathbf{x}_{\mathbf{i}}^{\prime}, t\right)$ focuses at $\mathbf{x}_{\mathbf{i}}{ }^{\prime}$ at $t=0$. At the focusing depth level $\partial D_{i}$ of $f_{1}$, we define $f_{1}\left(\mathbf{x}, \mathbf{x}_{\mathbf{i}}^{\prime}, t\right)$ as $\delta\left(\mathbf{x}_{H}-\mathbf{x}_{H}^{\prime}\right) \delta(t)$, a 2D and 1D Dirac delta function in space and time, respectively (see Figure $2 \mathrm{~b}$ and Table 1). Below the focusing depth level, $f_{1}\left(\mathbf{x}, \mathbf{x}_{\mathbf{i}}^{\prime}, t\right)$ continues to diverge as a downgoing field $f_{1}^{+}\left(\mathbf{x}, \mathbf{x}_{\mathbf{i}}^{\prime}, t\right)$ into the reflection-free reference half-space.

Similarly, Table 2 and Figure $2 \mathrm{~b}$ show the one-way wavefields for the $f_{2}$ function at the depth levels $\partial D_{0}$ and $\partial D_{i}$.

In this case, $f_{2}^{-}\left(\mathbf{x}, \mathbf{x}_{\mathbf{0}}^{\prime \prime}, t\right)$ is shaped such that the function $f_{2}\left(\mathbf{x}, \mathbf{x}_{\mathbf{0}}^{\prime \prime}, t\right)$ focuses at $\mathbf{x}_{\mathbf{0}}^{\prime \prime}$ at $t=0$. At the focusing depth level $\partial D_{0}$ of $f_{2}$, we define $f_{2}\left(\mathbf{x}, \mathbf{x}_{\mathbf{0}}^{\prime \prime}, t\right)$ as $\delta\left(\mathbf{x}_{H}-\mathbf{x}_{H}^{\prime \prime}\right) \delta(t)$ (see Figure $2 \mathrm{~b}$ and Table 2). After focusing, $f_{2}\left(\mathbf{x}, \mathbf{x}_{\mathbf{0}}^{\prime \prime}, t\right)$ continues to diverge as an upgoing field $f_{2}^{-}\left(\mathbf{x}, \mathbf{x}_{\mathbf{0}}^{\prime \prime}, t\right)$ into the homogeneous upper half-space.

The focusing functions are independent of the surface boundary condition of the actual medium because these functions reside only in the reference medium. If the media were homogeneous, the focusing function $f_{2}$ would consist of the time-reversed direct wave between the focal point and $\partial D_{i}$. However, in an inhomogeneous medium, the focusing function $f_{2}$ consists of the time-reversed direct wave and the coda $M$ following the time-reversed direct wave. The coda $M$ consists of the scattered waves that result when the time-reversed direct wave transmits through the medium to the focus point.

a)

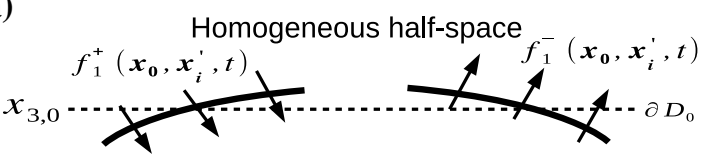

Actual inhomogeneous

$D$

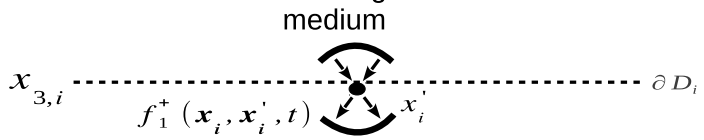

Reflection-free reference half-space

b)

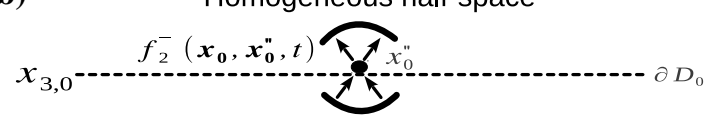

Actual inhomogeneous

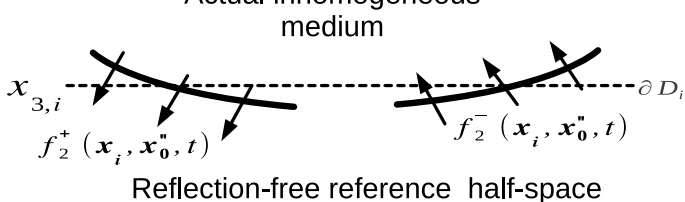

Figure 2. (a) Focusing function $f_{1}$ that focuses at $\mathbf{x}_{\mathbf{i}}^{\prime}$ and (b) focusing function $f_{2}$ that focuses at $\mathbf{x}_{\mathbf{0}}^{\prime \prime}$.
In the frequency domain, the focusing function $f_{2}$ is related to the Green's function $G_{0}$ of the actual inhomogeneous medium without a free surface by (Wapenaar et al., 2013a)

$$
\begin{aligned}
G_{0}\left(\mathbf{x}_{\mathbf{i}}^{\prime}, \mathbf{x}_{\mathbf{0}}^{\prime \prime}, \omega\right)= & f_{2}\left(\mathbf{x}_{\mathbf{i}}^{\prime}, \mathbf{x}_{\mathbf{0}}^{\prime \prime}, \omega\right)^{*} \\
& +\int_{\partial D_{0}} f_{2}\left(\mathbf{x}_{\mathbf{i}}^{\prime}, \mathbf{x}_{\mathbf{0}}, \omega\right) R_{0}\left(\mathbf{x}_{\mathbf{0}}, \mathbf{x}_{\mathbf{0}}^{\prime \prime}, \omega\right) d \mathbf{x}_{\mathbf{0}},
\end{aligned}
$$

where * represents the complex conjugate. In a situation without the free surface, we ignore the downward-reflected waves at the acquisition level, i.e., the free surface multiples. In the actual inhomogeneous medium, in the presence of a free surface, we account for the waves reflecting from the free surface (details are given in Appendix A, as shown in equation A-5). For this situation, the one-way wavefields are shown in Table 3 and Figure 3. We define wave state $B$ as the acoustic state with the wavefields of the actual medium (Wapenaar et al., 2013a).

We can use the one-way reciprocity theorems of the convolution and correlation type for flux-normalized one-way wavefields (Wapenaar and Grimbergen, 1996):

Table 1. One-way wavefields of the focusing function $f_{1}$ at the acquisition surface $\partial D_{0}$ and the level where $f_{1}$ focuses $\partial D_{i}$. The symbol $p_{A}^{ \pm}$represents one-way wavefields in the frequency domain, at arbitrary depth levels in the reference medium (see Figure 2a).

\begin{tabular}{ll} 
On $\partial D_{0}:$ & $p_{A}^{+}=f_{1}^{+}\left(\mathbf{x}_{\mathbf{0}}, \mathbf{x}_{\mathbf{i}}^{\prime}, \omega\right)$ \\
& $p_{A}^{-}=f_{1}^{-}\left(\mathbf{x}_{\mathbf{0}}, \mathbf{x}_{\mathbf{i}}^{\prime}, \omega\right)$ \\
On $\partial D_{i}:$ & $p_{A}^{+}=f_{1}^{+}\left(\mathbf{x}_{\mathbf{i}}, \mathbf{x}_{\mathbf{i}}^{\prime}, \omega\right)=\delta\left(\mathbf{x}_{H}-\mathbf{x}_{H}^{\prime}\right)$ \\
& $p_{A}^{-}=f_{1}^{-}\left(\mathbf{x}_{\mathbf{i}}, \mathbf{x}_{\mathbf{i}}^{\prime}, \omega\right)=0$ \\
\hline
\end{tabular}

Table 2. One-way wavefields of the focusing function $f_{2}$ at the depth level $\partial D_{0}$ and $\partial D_{i}$. The symbol $p_{A}^{ \pm}$represents oneway wavefields in the frequency domain, at arbitrary depth levels in the reference medium (see Figure 2b).

On $\partial D_{0}$ :

$$
\begin{aligned}
& p_{A}^{+}=f_{2}^{+}\left(\mathbf{x}_{\mathbf{0}}, \mathbf{x}_{\mathbf{0}}^{\prime \prime}, \omega\right)=0 \\
& p_{A}^{-}=f_{2}^{-}\left(\mathbf{x}_{\mathbf{0}}, \mathbf{x}_{\mathbf{0}}^{\prime \prime}, \omega\right)=\delta\left(\mathbf{x}_{H}-\mathbf{x}_{H}^{\prime \prime}\right) \\
& p_{A}^{+}=f_{2}^{+}\left(\mathbf{x}_{\mathbf{i}}, \mathbf{x}_{\mathbf{0}}^{\prime \prime}, \omega\right) \\
& p_{A}^{-}=f_{2}^{-}\left(\mathbf{x}_{\mathbf{i}}, \mathbf{x}_{\mathbf{0}}^{\prime \prime}, \omega\right)
\end{aligned}
$$

On $\partial D_{i}$ :

Table 3. One-way wavefields in the actual inhomogeneous medium in the presence of a free surface at the depth level $\partial D_{0}$ and $\partial D_{i}$. The symbol $p_{B}^{ \pm}$represents one-way wavefields at arbitrary depth levels in the inhomogeneous medium, whereas $r$ is the reflection coefficient of the free surface (see Figure 3).

On $\partial D_{0}: \quad p_{B}^{+}=G^{+}\left(\mathbf{x}_{\mathbf{0}}, \mathbf{x}_{\mathbf{0}}^{\prime \prime}, \omega\right)=\delta\left(\mathbf{x}_{\mathbf{H}}-\mathbf{x}_{\mathbf{H}}^{\prime \prime}\right)+r R\left(\mathbf{x}_{\mathbf{0}}, \mathbf{x}_{\mathbf{0}}^{\prime \prime}, \omega\right)$ $p_{B}^{-}=G^{-}\left(\mathbf{x}_{\mathbf{0}}, \mathbf{x}_{\mathbf{0}}^{\prime \prime}, \omega\right)=R\left(\mathbf{x}_{\mathbf{0}}, \mathbf{x}_{\mathbf{0}}^{\prime \prime}, \omega\right)$

On $\partial D_{i}: \quad p_{B}^{+}=G^{+}\left(\mathbf{x}_{\mathbf{i}}, \mathbf{x}_{\mathbf{0}}^{\prime \prime}, \omega\right)$ $p_{B}^{-}=G^{-}\left(\mathbf{x}_{\mathbf{i}}, \mathbf{x}_{\mathbf{0}}^{\prime \prime}, \omega\right)$ 


$$
\begin{gathered}
\int_{\partial D_{0}}\left[p_{A}^{+} p_{B}^{-}-p_{A}^{-} p_{B}^{+}\right] d \mathbf{x}_{\mathbf{0}}=\int_{\partial D_{i}}\left[p_{A}^{+} p_{B}^{-}-p_{A}^{-} p_{B}^{+}\right] d \mathbf{x}_{\mathbf{i}}, \\
\int_{\partial D_{0}}\left[\left(p_{A}^{+}\right)^{*} p_{B}^{+}-\left(p_{A}^{-}\right)^{*} p_{B}^{-}\right] d \mathbf{x}_{\mathbf{0}}=\int_{\partial D_{i}}\left[\left(p_{A}^{+}\right)^{*} p_{B}^{+}-\left(p_{A}^{-}\right)^{*} p_{B}^{-}\right] d \mathbf{x}_{\mathbf{i}},
\end{gathered}
$$

respectively, to relate the one-way wavefields between the two focusing functions $\left(f_{1}\right.$ and $\left.f_{2}\right)$ and the one-way wavefields between the focusing functions and the actual medium. Here, $p_{A}^{ \pm}$and $p_{B}^{ \pm}$are the one-way wavefields of the focusing functions and the actual medium, respectively, in the frequency domain. As shown in Appendix A, the corresponding two-way Green's function in the presence of the free surface is related to the focusing function $f_{2}$ by

$$
\begin{aligned}
G\left(\mathbf{x}_{\mathbf{i}}^{\prime}, \mathbf{x}_{\mathbf{0}}^{\prime \prime}, \omega\right)= & f_{2}\left(\mathbf{x}_{\mathbf{i}}^{\prime}, \mathbf{x}_{\mathbf{0}}^{\prime \prime}, \omega\right)^{*} \\
& +\int_{\partial D_{0}} f_{2}\left(\mathbf{x}_{\mathbf{i}}^{\prime}, \mathbf{x}_{\mathbf{0}}, \omega\right) R\left(\mathbf{x}_{\mathbf{0}}, \mathbf{x}_{\mathbf{0}}^{\prime \prime}, \omega\right) d \mathbf{x}_{\mathbf{0}} \\
& +r \int_{\partial D_{0}} f_{2}\left(\mathbf{x}_{\mathbf{i}}^{\prime}, \mathbf{x}_{\mathbf{0}}, \omega\right)^{*} R\left(\mathbf{x}_{\mathbf{0}}, \mathbf{x}_{\mathbf{0}}^{\prime \prime}, \omega\right) d \mathbf{x}_{\mathbf{0}},
\end{aligned}
$$

where, for simplicity, we assume that the reflection coefficient of the free surface satisfies $r=-1$. The two-way Green's function is defined as the superposition of the down- and upgoing fields, according to

$$
G\left(\mathbf{x}, \mathbf{x}_{\mathbf{0}}^{\prime \prime}, \omega\right)=G^{+}\left(\mathbf{x}, \mathbf{x}_{\mathbf{0}}^{\prime \prime}, \omega\right)+G^{-}\left(\mathbf{x}, \mathbf{x}_{\mathbf{0}}^{\prime \prime}, \omega\right) .
$$

Using reciprocity, the Green's functions on the left side of equations 4 and 6 can be interpreted as the response to a virtual source at $\mathbf{x}_{\mathbf{i}}^{\prime}$ for the situation without and with a free surface, respectively. To yield equations 4 and 6 , we use the one-way reciprocity relations (details are given in Appendix A for the retrieval of $G$, equation A-5). Note that the upgoing Green's function $G^{-}$in the actual inhomogeneous medium at $\partial D_{0}$ is the reflection response $R$ for a downward-radiating source at $\partial D_{0}$.

Equation 6 differs from equation 4 in two ways: First, the last term on the right side of equation 6 accounts for the waves that are reflected off the free surface. Second, equation 6 contains

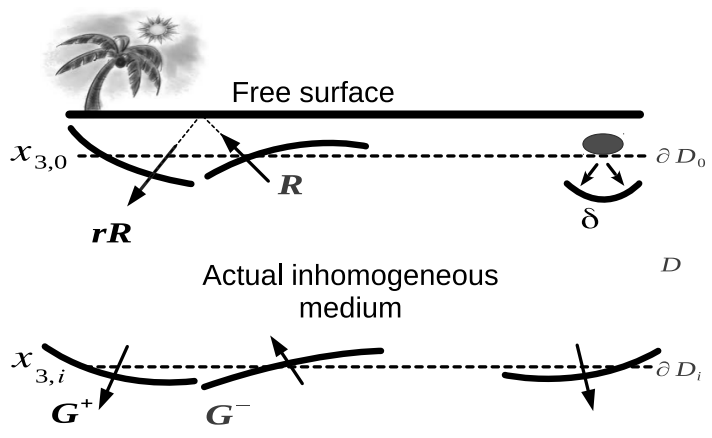

Actual inhomogeneous half-space

Figure 3. One-way Green's functions in the actual inhomogeneous medium in the presence of a free surface at the acquisition surface $\partial D_{0}$ and the arbitrary surface $\partial D_{i}$. These are the quantities that are used in the reciprocity theorems. The tree indicates the presence of the free surface. the reflection response $R$ for a medium with a free surface, whereas expression 4 contains the reflection response $R_{0}$ for a medium without a free surface.

Similar to our treatment of the focusing function $f_{2}$, we can define another focusing function $g_{2}$, such that

$$
g_{2}\left(\mathbf{x}, \mathbf{x}_{\mathbf{0}}^{\prime \prime}, \omega\right)=f_{2}^{+}\left(\mathbf{x}, \mathbf{x}_{\mathbf{0}}^{\prime \prime}, \omega\right)-f_{2}^{-}\left(\mathbf{x}, \mathbf{x}_{\mathbf{0}}^{\prime \prime}, \omega\right) .
$$

We use $g_{2}$ to obtain a difference Green's function $\tilde{G}$, which is similar to expression 6 :

$$
\begin{aligned}
\tilde{G}\left(\mathbf{x}_{\mathbf{i}}^{\prime}, \mathbf{x}_{\mathbf{0}}^{\prime \prime}, \omega\right)= & -g_{2}\left(\mathbf{x}_{\mathbf{i}}^{\prime}, \mathbf{x}_{\mathbf{0}}^{\prime \prime}, \omega\right)^{*} \\
& +\int_{\partial D_{0}} g_{2}\left(\mathbf{x}_{\mathbf{i}}^{\prime}, \mathbf{x}_{\mathbf{0}}, \omega\right) R\left(\mathbf{x}_{\mathbf{0}}, \mathbf{x}_{\mathbf{0}}^{\prime \prime}, \omega\right) d \mathbf{x}_{\mathbf{0}} \\
& -r \int_{\partial D_{0}} g_{2}\left(\mathbf{x}_{\mathbf{i}}^{\prime}, \mathbf{x}_{\mathbf{0}}, \omega\right)^{*} R\left(\mathbf{x}_{\mathbf{0}}, \mathbf{x}_{\mathbf{0}}^{\prime \prime}, \omega\right) d \mathbf{x}_{\mathbf{0}} .
\end{aligned}
$$

We call $\tilde{G}$ the difference Green's function because

$$
\tilde{G}\left(\mathbf{x}_{\mathbf{i}}^{\prime}, \mathbf{x}_{\mathbf{0}}^{\prime \prime}, \omega\right)=G^{+}\left(\mathbf{x}_{\mathbf{i}}^{\prime}, \mathbf{x}_{\mathbf{0}}^{\prime \prime}, \omega\right)-G^{-}\left(\mathbf{x}_{\mathbf{i}}^{\prime}, \mathbf{x}_{\mathbf{0}}^{\prime \prime}, \omega\right)
$$

and it is used to obtain the up- and downgoing Green's functions by combining it with $G$ in equation 7 .

To yield the upgoing Green's function, we subtract equations 7 and 10 :

$$
G^{-}\left(\mathbf{x}_{\mathbf{i}}^{\prime}, \mathbf{x}_{\mathbf{0}}^{\prime \prime}, \omega\right)=\frac{1}{2}\left[G\left(\mathbf{x}_{\mathbf{i}}^{\prime}, \mathbf{x}_{\mathbf{0}}^{\prime \prime}, \omega\right)-\tilde{G}\left(\mathbf{x}_{\mathbf{i}}^{\prime}, \mathbf{x}_{\mathbf{0}}^{\prime \prime}, \omega\right)\right] .
$$

Similarly, we obtain the downgoing Green's function by adding equations 6 and 10

$$
G^{+}\left(\mathbf{x}_{\mathbf{i}}^{\prime}, \mathbf{x}_{\mathbf{0}}^{\prime \prime}, \omega\right)=\frac{1}{2}\left[G\left(\mathbf{x}_{\mathbf{i}}^{\prime}, \mathbf{x}_{\mathbf{0}}^{\prime \prime}, \omega\right)+\tilde{G}\left(\mathbf{x}_{\mathbf{i}}^{\prime}, \mathbf{x}_{\mathbf{0}}^{\prime \prime}, \omega\right)\right] .
$$

These up- and downgoing $\left(G^{-}\right.$and $\left.G^{+}\right)$Green's functions at the focal point are used for imaging (Marchenko imaging) and include primaries and all multiples. Up- and downgoing Green's functions have been used for imaging the subsurface (Wapenaar et al., 2011; Behura et al., 2012; Broggini et al., 2012, 2014). However, their Green's functions contain only primaries and internal multiples. In this paper, the up- and downgoing Green's functions also include free-surface multiples.

The use of up- and downgoing wavefields for imaging is not a new principle. Claerbout (1971), Wapenaar et al. (2000), and Amundsen (2001) show that one can get the reflection response below an arbitrary depth level once the up- and downgoing wavefields are available. The governing equation that relates the up- and downgoing Green's function is

$$
\begin{aligned}
& G^{-}\left(\mathbf{x}_{\mathbf{i}}^{\prime}, \mathbf{x}_{\mathbf{0}}^{\prime \prime}, t\right) \\
& \quad=\int_{\partial D_{i}} d \mathbf{x}_{\mathbf{i}} \int_{-\infty}^{\infty} G^{+}\left(\mathbf{x}_{\mathbf{i}}, \mathbf{x}_{\mathbf{0}}^{\prime \prime}, t-t^{\prime}\right) R_{0}\left(\mathbf{x}_{\mathbf{i}}^{\prime}, \mathbf{x}_{\mathbf{i}}, t^{\prime}\right) d t^{\prime},
\end{aligned}
$$

where $\partial D_{i}$ is an arbitrary depth level and $R_{0}$ is the reflection response of the medium below $\partial D_{i}$. In addition, $R_{0}$ at $\partial D_{i}$ contains no reflections from above this depth level. We can think of $R_{0}$ as the reflection response of a truncated medium, where the truncated 
medium is the same as the true medium below $\partial D_{i}$ and reflection free above. Equation 13 states that $G^{-}$is represented by the convolution of $G^{+}$with $R_{0}$ and integration along all source positions $\mathbf{x}^{\prime}$ of $R_{0}$.

We solve for $R_{0}$ by MDD (Van der Neut et al., 2011) because the time integral is a convolution. The subsurface image is subsequently obtained by taking the zero lag of $R_{0}$, i.e., taking $t=0$ at each depth level in the model and at zero offset, $\mathbf{x}_{\mathbf{i}}=\mathbf{x}_{\mathbf{i}}^{\prime}$ (for each $\partial D_{i}$ ); this is called the zero-offset imaging condition. Alternatively, once we obtain $R_{0}$ at an arbitrary $\partial D_{i}$, we can also apply a standard imaging procedure, for instance, downward continuation, to image below $\partial D_{i}$. This is because $R_{0}$ is the reflection response of the truncated medium below $\partial D_{i}$ for sources and receivers at $\partial D_{i}$.

\section{NUMERICAL EXAMPLES}

We use three numerical examples that show the retrieval of the Green's functions and imaging using the associated Green's functions.

We first consider a 1D model that has a high impedance layer generic to salt models as shown in Figure 4. A receiver at the surface records the reflected waves. To retrieve the Green's function in 1D, one needs the traveltime of the first arriving wave from the virtual source to the surface. The traveltime of the first arriving wave is used to temporally separate the Green's function from the focusing solution $f_{2}$ in the time-domain representation of equation 6 . To obtain the focusing function $f_{2}$, we evaluate expression 6 for a time earlier than the first arriving wave and setting the left side of equation 6 to zero. The remaining expression is an equation for $f_{2}$, which is solved iteratively (details are given in Appendix A, equations A-6-A-10).

Once the focusing solution has been found (see Figure 5), it is substituted in equation 6 to retrieve the Green's function (for this example, the Green's function is the response to the virtual source at a depth of $2.75 \mathrm{~km}$, [dot in Figure 4], recorded at the surface; Figure 6). This Green's function $G$, arbitrarily scaled to its maximum amplitude (see Figure 6), is the response at the surface $\partial D_{0}$ to the virtual source.

We also model the Green's function using finite differences to verify that the Green's function retrieved from our algorithm is accurate, and we superimposed this result on Figure 6 . The vertical scale of Figure 6 is enlarged to better illustrate the model and retrieved Green's function. For this reason, the first arrival at time

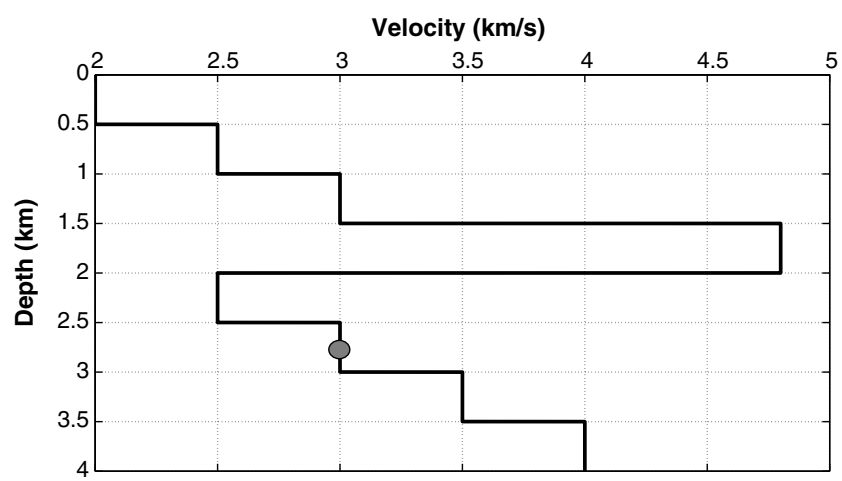

Figure 4. Velocity model with high impedance layer at $1.5 \mathrm{~km}$; the dot is the position of the virtual source.
$1.0 \mathrm{~s}$ is clipped. The difference between the modeled and the retrieved Green's function is negligible relative to the average amplitude of the Green's function, as seen in Figure 6. The arrivals caused by the free surface are shown in Figure 7 compared with the arrivals in the absence of a free surface.

The corresponding image of the model in Figure 4 shown in Figure 8 illustrates the correct location of the reflectors, as well as the correct scaled reflection coefficient. In 1D, the image we obtain is the deconvolution of the up- and downgoing Green's function for $t=0$ at each image point (Claerbout, 1985). There are some anomalous amplitudes in the Marchenko image (especially at approximately $200 \mathrm{~m}$ ), but they are small compared with the actual reflectors' amplitude and are attributed to the deconvolution imaging condition.

The model for the next example is taken from Weglein and Dragoset (2007), where the second primary event cancels with

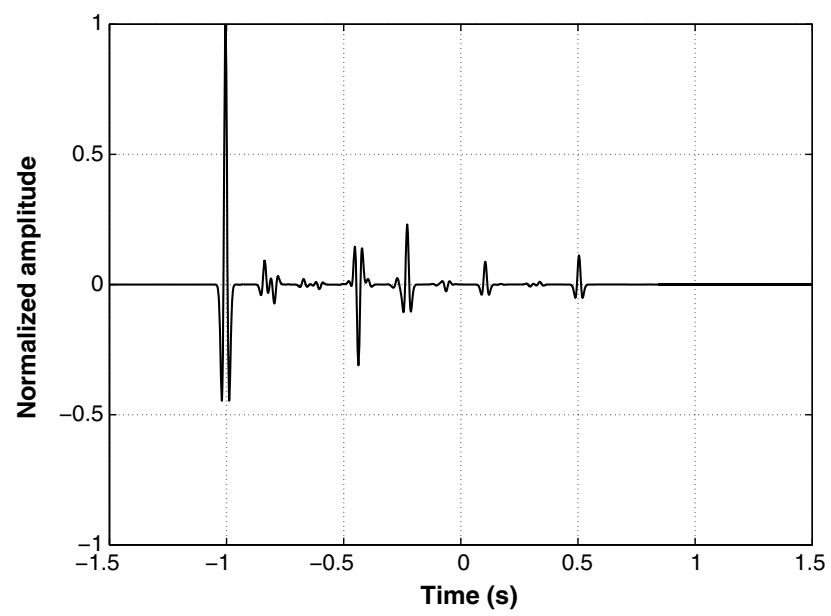

Figure 5. Focusing function $f_{2}$ at depth $2.75 \mathrm{~km}$ for the velocity model in Figure 4.

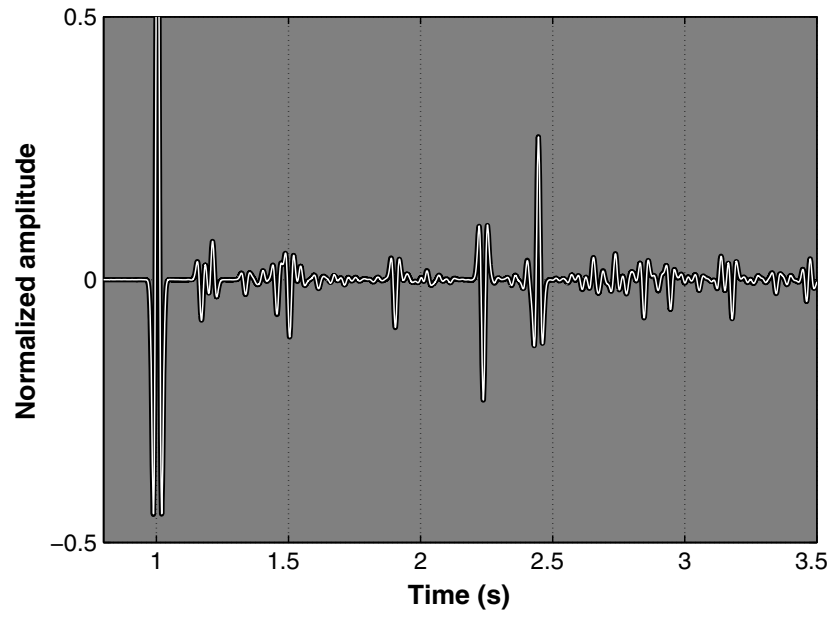

Figure 6. Retrieved Green's function (normalized by maximum amplitude) $G$ from a depth of $2.75 \mathrm{~km}$ to the surface (white). The modeled Green's function is displayed (in black) in the background. The Green's functions are associated to the model in Figure 4. 
the free-surface multiple from the first reflector. We demonstrate with this numerical example the retrieval of the Green's function (as well as its associated up- and downgoing Green's function)

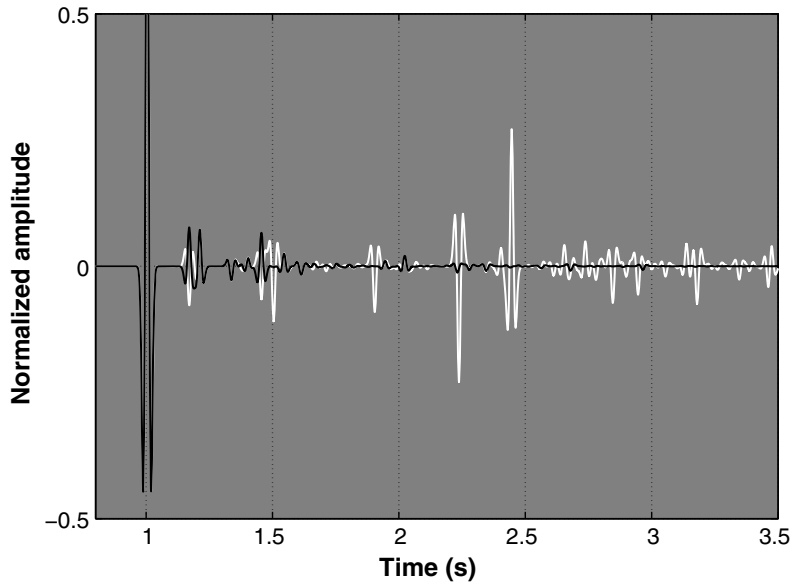

Figure 7. Impact of the free surface. The black line shows the Green's function in a medium without a free surface $G_{0}$, and the white line is the difference between $G$ and $G_{0}$; therefore, the white line shows events that are caused by the presence of the free surface.

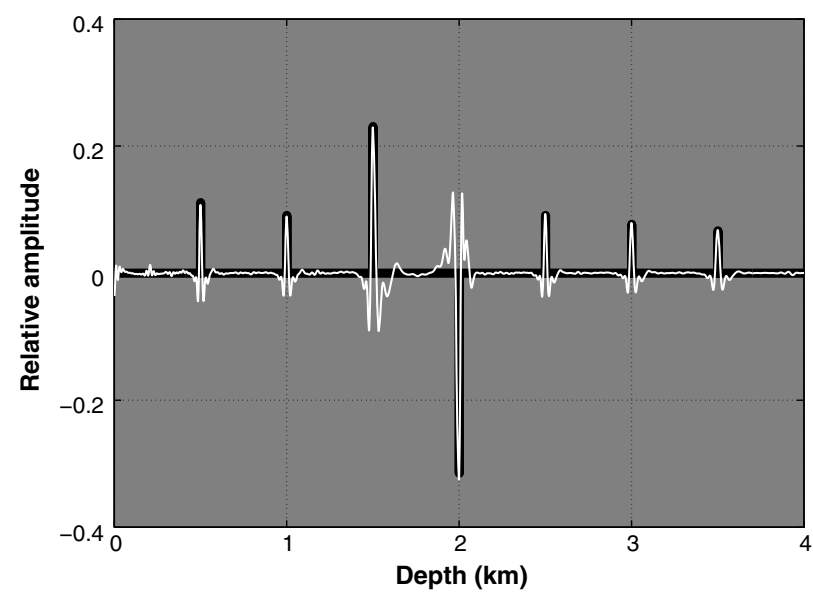

Figure 8. Marchenko imaging of Figure 4 in white, with the true reflectivity (in black) in the background.

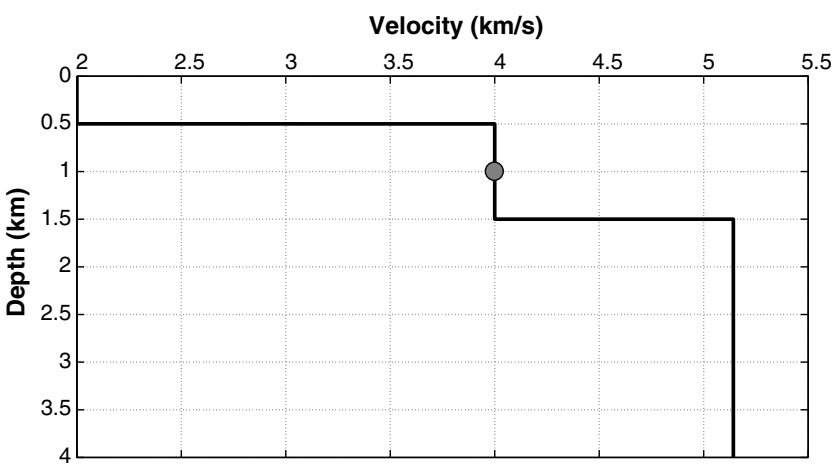

Figure 9. Simple velocity model, where the dot indicates the position of one virtual source at depth $1000 \mathrm{~m}$. at depth $1000 \mathrm{~m}$ for the model shown in Figure 9. Figure 10 illustrates some of the reflected events corresponding to this model. The associated reflected waves at the acquisition level, shown in Figure 11, are recorded $5 \mathrm{~m}$ below the free surface. As is shown in Figures 11 and 10, the second primary event $P_{2}$ is canceled by the free-surface multiple $F_{1}$ at $1.0 \mathrm{~s}$, and the other events (internal multiples and free surface multiples) interfere destructively with each other at later times.

The Green's function for a source at depth $1000 \mathrm{~m}$ is shown in Figure 12. The corresponding up- and downgoing Green's function
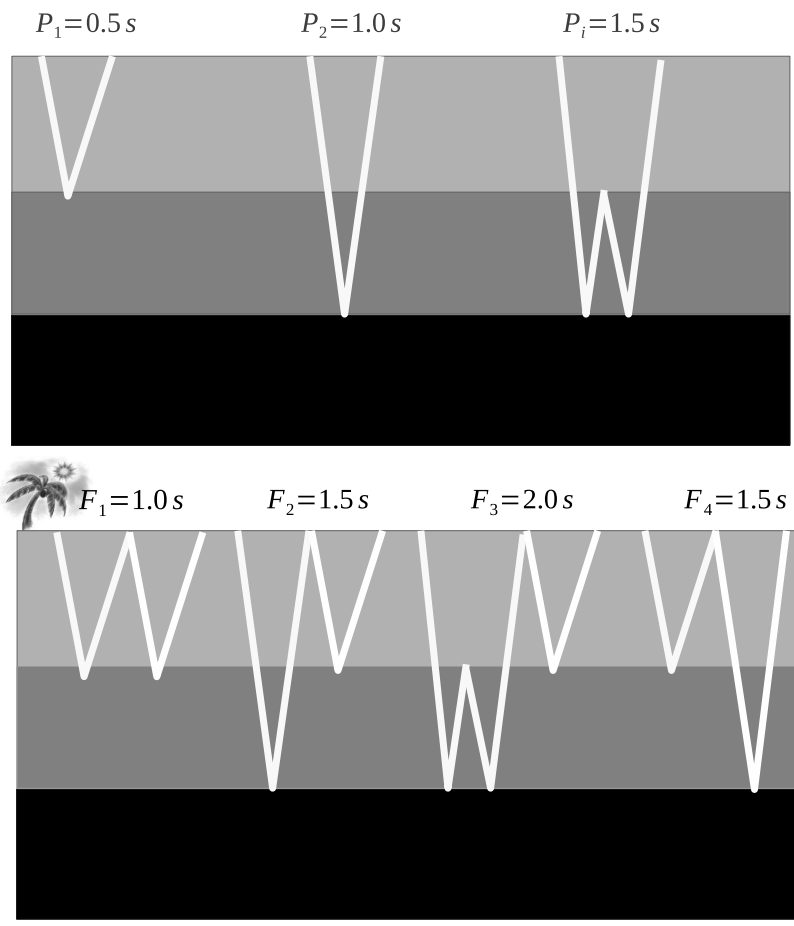

Figure 10. Sketch of some events that occur in the velocity model in Figure 9.

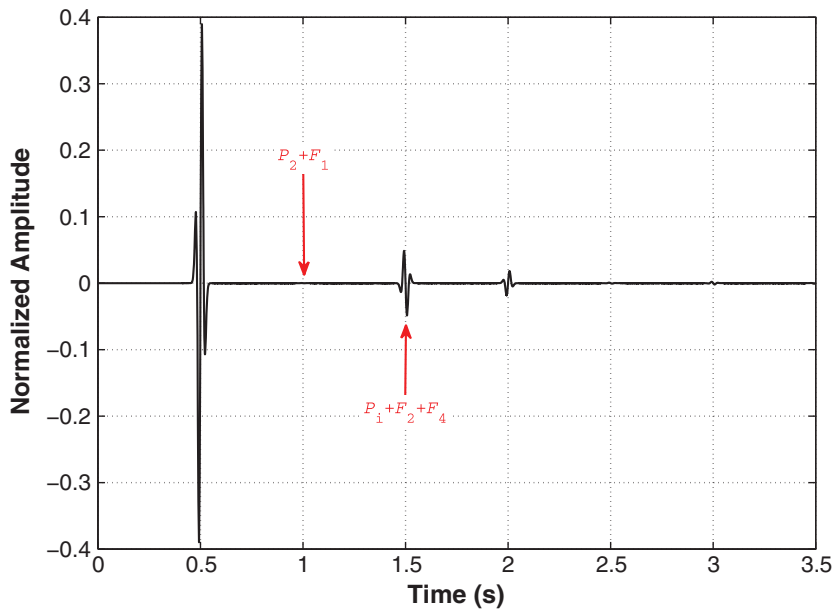

Figure 11. Reflection response with the direct waves removed (the events are scaled by the direct wave magnitude); the labels are referenced to in Figure 10. 
at depth $1000 \mathrm{~m}$ is illustrated in Figures 13 and 14, respectively. The computed traveltimes for this simple model of the up- and downgoing Green's function in Figure 15 correspond to the traveltimes of the events in the retrieved up- and downgoing Green's functions, hence confirming our decomposition of the Green's function into its associated up- and downgoing wavefields.

Figure 12 has an event at $0.6 \mathrm{~s}$, which is the reflection of the virtual source field from the second layer recorded at the surface. Hence, the Green's function retrieval algorithm correctly detects the missing reflector in the recorded waves. In the 1D model considered here, equation 13 , reduces to the deconvolution of the upgoing Green's function with the downgoing Green's function at every point in the velocity model yielding the correct positioning of the reflectors (see Figure 16), as well as the correct scaled reflection coefficient. Furthermore, the image is free of artifacts originating from surface-related and internal multiples. Importantly, the differ-

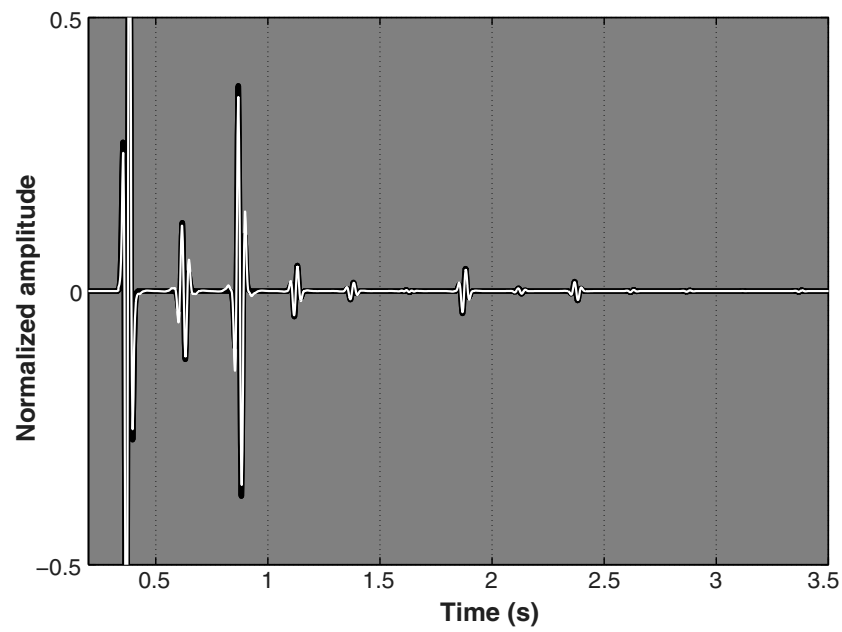

Figure 12. Green's function with virtual source at depth $1000 \mathrm{~m}$ and recording at the surface. The black thicker line is the modeled Green's function; superimposed on it is the retrieved Green's function. The plot limits are chosen between 0.5 and -0.5 normalized amplitude to better visualize the smaller amplitude events.

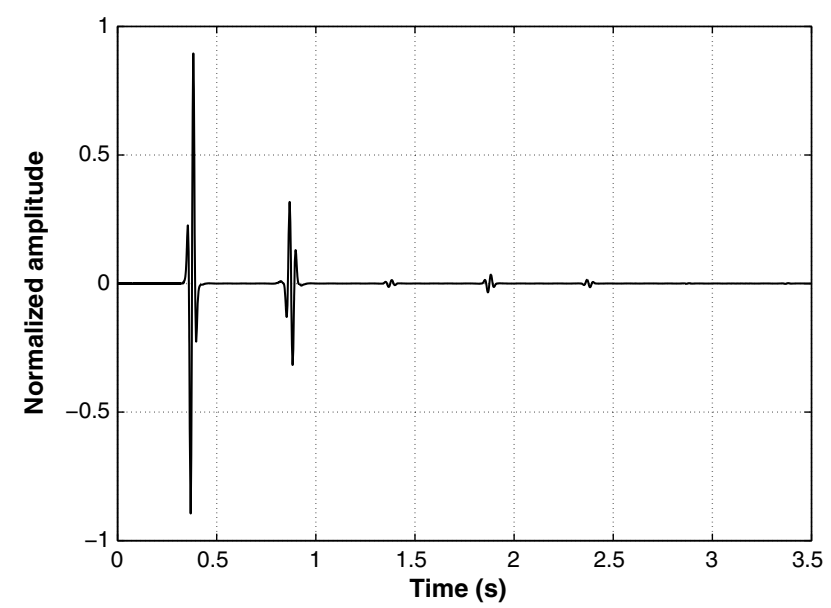

Figure 13. The Green's function for upgoing waves at the virtual source location $(1000 \mathrm{~m})$ and recording at the surface for the velocity in model in Figure 9. ence between the analysis of Weglein and Dragoset (2007) and our work is that they remove the multiples at the surface, whereas we derive the Green's function for a virtual source in the subsurface and, subsequently, a multiple-free image.

\section{DISCUSSION}

The new feature of our Green's function retrieval scheme compared with the earlier presented schemes is that we include the freesurface multiples in our Green's function. Hence, we do not need to remove the free-surface multiples from our reflection response. For our 1D numerical examples, we can identify the events that are caused by the presence of the free surface (the black line in Figure 7) by computing the difference of $G$ and $G_{0}$.

The arrivals that are caused by the free surface (black line in Figure 7) have higher amplitude and greater waveform complexity

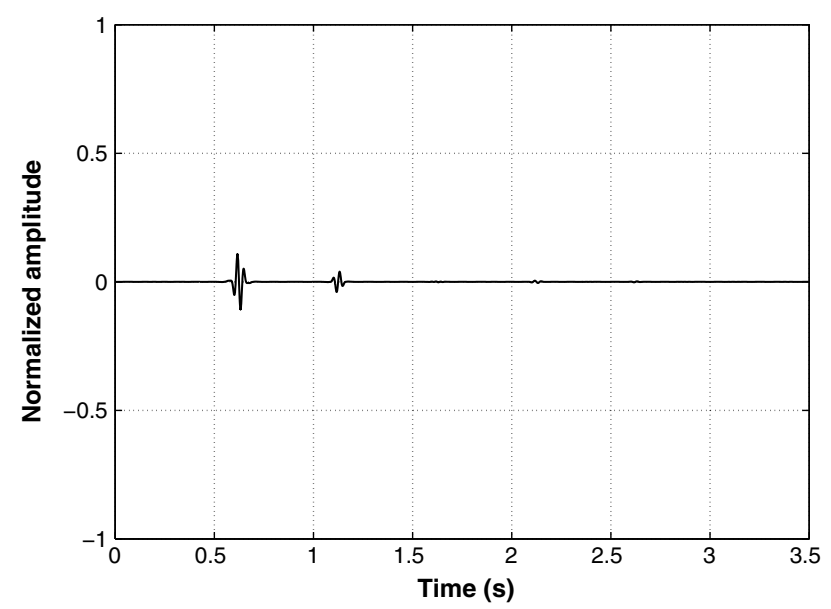

Figure 14. The Green's function for downgoing waves at the virtual source location $(1000 \mathrm{~m})$ and recording at the surface for the velocity in model in Figure 9.

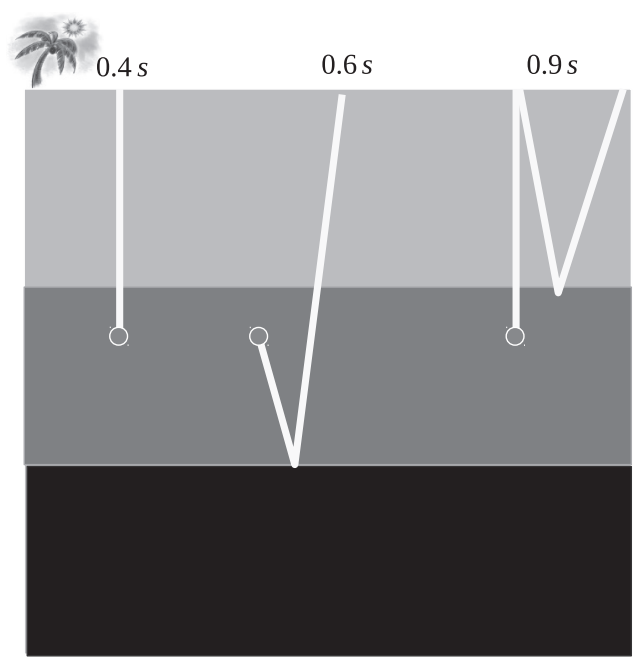

Figure 15. Sketch of some events that are present in the Green's function from the virtual source (gray dot) at $1000 \mathrm{~m}$ and recorded at the surface for the velocity in model in Figure 9. 


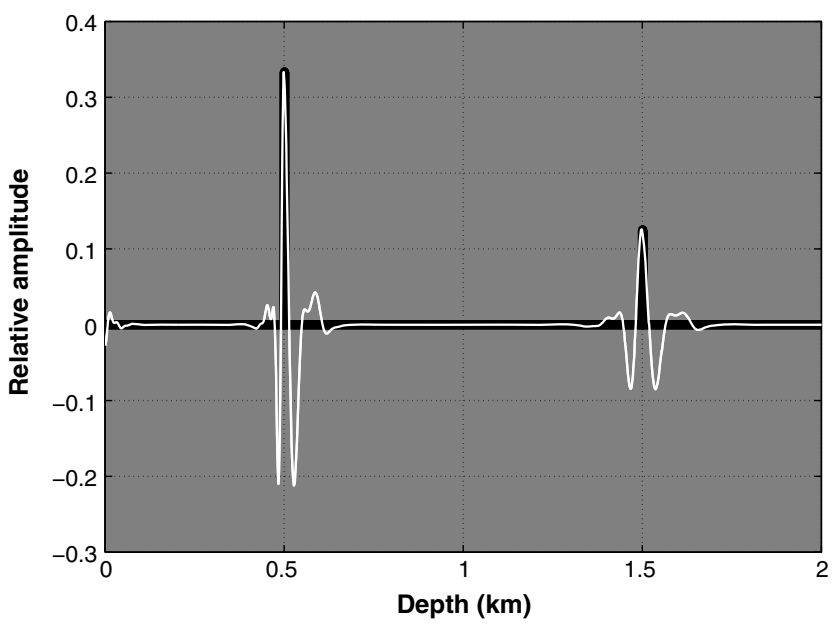

Figure 16. Image of the velocity model (Figure 9) after Marchenko imaging with the reflectivity overlain (in black).

compared with the events in the response without a free surface $G_{0}$ (white line in 7). This supports our conjecture that using the additional events and energy that free-surface reflections provide can benefit imaging of the subsurface, i.e., imaging using $G$ rather than $G_{0}$.

In our scheme to retrieve the Green's function, we require that the wavelet is removed from the reflection response (which can be done by deconvolution). In addition, we assume that the reflection response is due to a downgoing source. However, in the marine case, the source is generally placed a few meters below the surface; therefore, there is not only a downgoing component of the source, but also an upgoing component. In such situations, we consider the source wavelet to also include the upgoing component of the source. Consequently, the wavelet with which we deconvolve the reflection response at the surface is no longer that of a monopole source but of a dipole source.

Although we show 1D examples, all equations are multidimensional. In $2 \mathrm{D}$ or $3 \mathrm{D}$ media, a smooth version of the slowness (1/velocity) can be used to get an estimate of the direct arriving wave from the virtual source to the surface, but the small-scale details of the velocity and density need not be known. The direct arriving wave can be obtained using finite-difference modeling of the waveforms. In $1 \mathrm{D}$, a velocity model is not necessary, unless we want a depth image as in the examples we show in this paper.

We have also briefly investigate Marchenko imaging when the reflection response is corrupted with noise. In such noisy cases, the noise level in the reflection response and the Marchenko image is similar although our Green's function retrieval algorithm is nonlinear. However, further investigation is needed to properly understand the accuracy of Marchenko imaging when the reflection response is corrupted with noise.

\section{CONCLUSION}

We have extended the retrieval of the Green's function to include the presence of a free surface. This function includes primaries, internal multiples, and now also free-surface multiples. Significantly, our proposed method does not require any surface-related multiple removal of the reflection response. The resulting images of the subsurface are free of any artifacts of the free surface multiples and internal multiples; this is because Marchenko imaging is a natural way to use primaries and multiples in imaging.

\section{ACKNOWLEDGMENTS}

This work was funded by the sponsor companies of the Consortium Project on Seismic Inverse Methods for Complex Structures. We are grateful to D. Witters for her help in preparing this manuscript. We thank J. Robertsson, M. Ravasi, and two anonymous reviewers for their critical feedback.

\section{APPENDIX A \\ GREEN'S FUNCTION RETRIEVAL IN THE PRESENCE OF THE FREE SURFACE}

We discuss our adaptation of the method of Wapenaar et al. (2013b) to account for the free-surface reflections. The focusing functions are the wavefields that focus, in time and space, at a point in the medium or at the surface (Figure $2 \mathrm{a}$ and $2 \mathrm{~b}$ ). These focusing functions exist in the reference medium, which is homogeneous above the depth level $\partial D_{0}$ and reflection free below $\partial D_{i}$.

The reciprocity theorems for one-way (up- and downgoing) wavefields are derived by Wapenaar and Grimbergen (1996) (see equations A-3 and A-4). We use the convolution-type and correlation-type reciprocity theorems to find relationships between our upand downgoing wavefields. As discussed by Wapenaar et al. (2014), we obtain a relationship between the focusing functions $f_{1}$ and $f_{2}$ by using their respective up- and downgoing waves at each depth level $\partial D_{0}$ and $\partial D_{i}$ with the convolution reciprocity theorem

$$
f_{1}^{+}\left(\mathbf{x}_{\mathbf{0}}^{\prime \prime}, \mathbf{x}_{\mathbf{i}}^{\prime}, \omega\right)=f_{2}^{-}\left(\mathbf{x}_{\mathbf{i}}^{\prime}, \mathbf{x}_{\mathbf{0}}^{\prime \prime}, \omega\right),
$$

and correlation reciprocity theorem

$$
-f_{1}^{-}\left(\mathbf{x}_{\mathbf{0}}^{\prime \prime}, \mathbf{x}_{\mathbf{i}}^{\prime}, \omega\right)^{*}=f_{2}^{+}\left(\mathbf{x}_{\mathbf{i}}^{\prime}, \mathbf{x}_{\mathbf{0}}^{\prime \prime}, \omega\right) .
$$

Our actual inhomogeneous model with a free surface above $\partial D_{0}$ is shown in Figure 3. As opposed to the model in Wapenaar et al. (2013a), which does not have a free surface, we consider the reflections from the free surface for a downgoing source similar to the work of Wapenaar et al. (2004). In Figure 3, we describe the wavefield in its up- and downgoing components. The downward-propagating component of the wavefield (Green's function) at $\partial D_{0}$ is $G^{+}\left(\mathbf{x}_{\mathbf{0}}, \mathbf{x}_{\mathbf{0}}^{\prime \prime}, \omega\right)=\delta\left(\mathbf{x}_{\mathbf{H}}-\mathbf{x}_{\mathbf{H}}^{\prime \prime}\right)+r R\left(\mathbf{x}_{\mathbf{0}}, \mathbf{x}_{\mathbf{0}}^{\prime \prime}, \omega\right)$, which includes (in the right side) the downward-going impulsive source and the reflection from the free surface. The downgoing source $\delta\left(\mathbf{x}_{\mathbf{H}}-\mathbf{x}_{\mathbf{H}}^{\prime \prime}\right)$ is a 2D Dirac delta, where $\mathbf{x}_{\mathbf{H}}^{\prime \prime}$ is the lateral position of the focal point of $f_{2}$.

Note $G^{+}$is the component of the Green's function that is propagating downward at $\mathbf{x}_{\mathbf{0}}$ for a downward radiating source at $\mathbf{x}_{\mathbf{0}}^{\prime \prime}$. In the case without the free surface, there are no reflections from the free surface; hence, $G_{0}^{+}\left(\mathbf{x}_{\mathbf{0}}, \mathbf{x}_{\mathbf{0}}^{\prime \prime}, \omega\right)=\delta\left(\mathbf{x}_{\mathbf{H}}-\mathbf{x}_{\mathbf{H}}^{\prime \prime}\right)$ because $r=0$. The upward-going propagating part of the Green's function $G^{-}$ at $\partial D_{0}$ is the reflection response $R\left(\mathbf{x}_{\mathbf{0}}, \mathbf{x}_{\mathbf{0}}^{\prime \prime}, \omega\right)$. We consider the up- and downgoing components of the Green's function at $\partial D_{i}$. The downgoing component is $G^{+}\left(\mathbf{x}_{\mathbf{i}}, \mathbf{x}_{\mathbf{0}}^{\prime \prime}, \omega\right)$, whereas the upgoing component is $G^{-}\left(\mathbf{x}_{\mathbf{i}}, \mathbf{x}_{\mathbf{0}}^{\prime \prime}, \omega\right)\left(G^{+}\right.$and $G^{-}$, respectively, Figure ). We use the convolution and correlation reciprocity theorems to find relationships for the one-way wavefields of $f_{1}$ as shown in Figure 2a, 
and the one-way wavefields of the Green's function in the actual medium are shown in Figure 3:

$$
\begin{aligned}
G^{-}\left(\mathbf{x}_{\mathbf{i}}^{\prime}, \mathbf{x}_{\mathbf{0}}^{\prime \prime}, \omega\right)= & \int_{\partial D_{0}}\left[f_{1}^{+}\left(\mathbf{x}_{\mathbf{0}}, \mathbf{x}_{\mathbf{i}}^{\prime}, \omega\right) R\left(\mathbf{x}_{\mathbf{0}}, \mathbf{x}_{\mathbf{0}}^{\prime \prime}, \omega\right)\right. \\
& \left.-r f_{1}^{-}\left(\mathbf{x}_{\mathbf{0}}, \mathbf{x}_{\mathbf{i}}^{\prime}, \omega\right) R\left(\mathbf{x}_{\mathbf{0}}, \mathbf{x}_{\mathbf{0}}^{\prime \prime}, \omega\right)\right] d \mathbf{x} \\
& -f_{1}^{-}\left(\mathbf{x}_{\mathbf{0}}^{\prime \prime}, \mathbf{x}_{\mathbf{i}}^{\prime}, \omega\right),
\end{aligned}
$$

and

$$
\begin{aligned}
G^{+}\left(\mathbf{x}_{\mathbf{i}}^{\prime}, \mathbf{x}_{\mathbf{0}}^{\prime \prime}, \omega\right)= & -\int_{\partial D_{0}}\left[f_{1}^{-}\left(\mathbf{x}_{\mathbf{0}}, \mathbf{x}_{\mathbf{i}}^{\prime}, \omega\right)^{*} R\left(\mathbf{x}_{\mathbf{0}}, \mathbf{x}_{\mathbf{0}}^{\prime \prime}, \omega\right)\right. \\
& \left.-r f_{1}^{+}\left(\mathbf{x}_{\mathbf{0}}, \mathbf{x}_{\mathbf{i}}^{\prime}, \omega\right)^{*} R\left(\mathbf{x}_{\mathbf{0}}, \mathbf{x}_{\mathbf{0}}^{\prime \prime}, \omega\right)\right] d \mathbf{x} \\
& +f_{1}^{+}\left(\mathbf{x}_{\mathbf{0}}^{\prime \prime}, \mathbf{x}_{\mathbf{i}}^{\prime}, \omega\right)^{*}
\end{aligned}
$$

Equations A-3 and A-4 are similar to the relation for the up- and downgoing Green's function in Wapenaar et al. (2014); however, equations A-3 and A-4 also account for the reflected waves from the free surface. These free-surface reflections are the expressions in equations A-3 and A-4 that are multiplied by $r$. The two-way Green's function is obtained by adding equations A-3 and A-4, as well as using equations 2, 3, A-1, and A-2:

$$
\begin{aligned}
G\left(\mathbf{x}_{\mathbf{i}}^{\prime}, \mathbf{x}_{\mathbf{0}}^{\prime \prime}, \omega\right)= & f_{2}\left(\mathbf{x}_{\mathbf{i}}^{\prime}, \mathbf{x}_{\mathbf{0}}^{\prime \prime}, \omega\right)^{*} \\
& +\int_{\partial D_{0}} f_{2}\left(\mathbf{x}_{\mathbf{i}}^{\prime}, \mathbf{x}_{\mathbf{0}}, \omega\right) R\left(\mathbf{x}_{\mathbf{0}}, \mathbf{x}_{\mathbf{0}}^{\prime \prime}, \omega\right) d \mathbf{x}_{\mathbf{0}} \\
& +r \int_{\partial D_{0}} f_{2}\left(\mathbf{x}_{\mathbf{i}}^{\prime}, \mathbf{x}_{\mathbf{0}}, \omega\right)^{*} R\left(\mathbf{x}_{\mathbf{0}}, \mathbf{x}_{\mathbf{0}}^{\prime \prime}, \omega\right) d \mathbf{x}_{\mathbf{0}} .
\end{aligned}
$$

We consider equation A-5, in time, for the interval $t<t_{d}\left(\mathbf{x}_{\mathbf{i}}^{\prime}, \mathbf{x}_{\mathbf{0}}^{\prime \prime}\right)$, where $t_{d}$ is the traveltime for the first arrival of $G$. No waves arrive before $t_{d}\left(\mathbf{x}_{\mathbf{i}}^{\prime}, \mathbf{x}_{\mathbf{0}}^{\prime \prime}\right)$ because $t_{d}$ is the time for the first-arriving event. Therefore, $G\left(\mathbf{x}_{\mathbf{i}}^{\prime}, \mathbf{x}_{\mathbf{0}}^{\prime \prime}, \omega\right)$ vanishes for $t<t_{d}\left(\mathbf{x}_{\mathbf{i}}^{\prime}, \mathbf{x}_{\mathbf{0}}^{\prime \prime}\right)$, and as a result,

$$
\begin{aligned}
0= & f_{2}\left(\mathbf{x}_{\mathbf{i}}^{\prime}, \mathbf{x}_{\mathbf{0}}^{\prime \prime},-t\right) \\
& +\int_{\partial D_{0}} d \mathbf{x}_{\mathbf{0}} \int_{-\infty}^{t} f_{2}\left(\mathbf{x}_{\mathbf{i}}^{\prime}, \mathbf{x}_{\mathbf{0}}, t^{\prime}\right) R\left(\mathbf{x}_{\mathbf{0}}, \mathbf{x}_{\mathbf{0}}^{\prime \prime}, t-t^{\prime}\right) d t^{\prime} \\
& +r \int_{\partial D_{0}} d \mathbf{x}_{\mathbf{0}} \int_{-t}^{\infty} f_{2}\left(\mathbf{x}_{\mathbf{i}}^{\prime}, \mathbf{x}_{\mathbf{0}}, t^{\prime}\right) R\left(\mathbf{x}_{\mathbf{0}}, \mathbf{x}_{\mathbf{0}}^{\prime \prime}, t+t^{\prime}\right) d t^{\prime}
\end{aligned}
$$

We use the same ansatz for $f_{2}$ as Wapenaar et al. (2013a) because we are using the same reference medium, i.e., the model where the focusing functions exist. The ansatz is given by

$$
f_{2}\left(\mathbf{x}_{\mathbf{i}}, \mathbf{x}_{\mathbf{0}}^{\prime \prime}, t\right)=\left[T_{d}\left(\mathbf{x}_{\mathbf{i}}, \mathbf{x}_{\mathbf{0}}^{\prime \prime}, t\right)\right]^{\text {inv }}+M\left(\mathbf{x}_{\mathbf{i}}, \mathbf{x}_{\mathbf{0}}^{\prime \prime}, t\right),
$$

where $\left[T_{d}\left(\mathbf{x}_{\mathbf{i}}, \mathbf{x}_{\mathbf{0}}^{\prime \prime}, t\right)\right]^{\text {inv }}$, defined as the inverse of the direct arrival of the transmission response, is the first-arriving event of $f_{2}\left(\mathbf{x}_{\mathbf{i}}, \mathbf{x}_{\mathbf{0}}^{\prime \prime}, t\right)$ and $M\left(\mathbf{x}_{\mathbf{i}}, \mathbf{x}_{\mathbf{0}}^{\prime \prime}, t\right)$ is the scattering coda of $f_{2}$ following the first arrival as shown by Wapenaar et al. (2013a). The substitution of expression A-7 in equation A-6 yields

$$
\begin{aligned}
0 & =M\left(\mathbf{x}_{\mathbf{i}}^{\prime}, \mathbf{x}_{\mathbf{0}}^{\prime \prime},-t\right) \\
& +\int_{\partial D_{0}} d \mathbf{x}_{\mathbf{0}} \int_{-\infty}^{-t_{d}^{\epsilon}\left(\mathbf{x}_{\mathbf{i}}^{\prime}, \mathbf{x}_{\mathbf{0}}\right)}\left[T_{d}\left(\mathbf{x}_{\mathbf{i}}^{\prime}, \mathbf{x}_{\mathbf{0}}, t^{\prime}\right)\right]^{i n v} R\left(\mathbf{x}_{\mathbf{0}}, \mathbf{x}_{\mathbf{0}}^{\prime \prime}, t-t^{\prime}\right) d t^{\prime} \\
& +\int_{\partial D_{0}} d \mathbf{x}_{\mathbf{0}} \int_{-t_{d}^{\epsilon}\left(\mathbf{x}_{\mathbf{i}}^{\prime}, \mathbf{x}_{\mathbf{0}}\right)}^{t} M\left(\mathbf{x}_{\mathbf{i}}^{\prime}, \mathbf{x}_{\mathbf{0}}, t^{\prime}\right) R\left(\mathbf{x}_{\mathbf{0}}, \mathbf{x}_{\mathbf{0}}^{\prime \prime}, t-t^{\prime}\right) d t^{\prime} \\
& +r \int_{\partial D_{0}} d \mathbf{x}_{\mathbf{0}} \int_{-t_{d}^{\epsilon}\left(\mathbf{x}_{\mathbf{i}}^{\prime}, \mathbf{x}_{\mathbf{0}}\right)}^{\infty} M\left(\mathbf{x}_{\mathbf{i}}^{\prime}, \mathbf{x}_{\mathbf{0}}, t^{\prime}\right) R\left(\mathbf{x}_{\mathbf{0}}, \mathbf{x}_{\mathbf{0}}^{\prime \prime}, t+t^{\prime}\right) d t^{\prime} \\
& +r \int_{\partial D_{0}} d \mathbf{x}_{\mathbf{0}} \int_{-t}^{-t_{d}^{\epsilon}\left(\mathbf{x}_{\mathbf{i}}^{\prime}, \mathbf{x}_{\mathbf{0}}\right)}\left[T_{d}\left(\mathbf{x}_{\mathbf{i}}^{\prime}, \mathbf{x}_{\mathbf{0}}, t^{\prime}\right)\right]^{i n v} R\left(\mathbf{x}_{\mathbf{0}}, \mathbf{x}_{\mathbf{0}}^{\prime \prime}, t+t^{\prime}\right) d t^{\prime},
\end{aligned}
$$

for $t<t_{d}\left(\mathbf{x}_{\mathbf{i}}^{\prime}, \mathbf{x}_{\mathbf{0}}^{\prime \prime}\right)$ with $t_{d}^{\epsilon}\left(\mathbf{x}_{\mathbf{i}}^{\prime}, \mathbf{x}_{\mathbf{0}}^{\prime \prime}\right)=t_{d}\left(\mathbf{x}_{\mathbf{i}}^{\prime}, \mathbf{x}_{\mathbf{0}}^{\prime \prime}\right)-\epsilon$, where $\epsilon$ is a small positive constant to include the direct arrival in the integral. Equation A-8 is a Fredholm integral of the second kind and can be solved iteratively as follows:

$$
\begin{aligned}
M_{k}\left(\mathbf{x}_{\mathbf{i}}^{\prime}, \mathbf{x}_{\mathbf{0}}^{\prime \prime},-t\right) \\
\quad=M_{0}\left(\mathbf{x}_{\mathbf{i}}^{\prime}, \mathbf{x}_{\mathbf{0}}^{\prime \prime},-t\right) \\
\quad-\int_{\partial D_{0}} d \mathbf{x}_{\mathbf{0}} \int_{-t_{d}^{e}\left(\mathbf{x}_{\mathbf{i}}^{\prime}, \mathbf{x}_{\mathbf{0}}\right)}^{\infty} M_{k-1}\left(\mathbf{x}_{\mathbf{i}}^{\prime}, \mathbf{x}_{\mathbf{0}}, t^{\prime}\right) R\left(\mathbf{x}_{\mathbf{0}}, \mathbf{x}_{\mathbf{0}}^{\prime \prime}, t-t^{\prime}\right) d t^{\prime} \\
\quad-r \int_{\partial D_{0}} d \mathbf{x}_{\mathbf{0}} \int_{-t_{d}^{e}\left(\mathbf{x}_{\mathbf{i}}^{\prime}, \mathbf{x}_{\mathbf{0}}\right)}^{\infty} M_{k-1}\left(\mathbf{x}_{\mathbf{i}}^{\prime}, \mathbf{x}_{\mathbf{0}}, t^{\prime}\right) R\left(\mathbf{x}_{\mathbf{0}}, \mathbf{x}_{\mathbf{0}}^{\prime \prime}, t+t^{\prime}\right) d t^{\prime},
\end{aligned}
$$

where

$$
\begin{aligned}
& M_{0}\left(\mathbf{x}_{\mathbf{i}}^{\prime}, \mathbf{x}_{\mathbf{0}}^{\prime \prime},-t\right) \\
& =-\int_{\partial D_{0}} d \mathbf{x}_{\mathbf{0}} \int_{-\infty}^{-t_{d}^{\epsilon}\left(\mathbf{x}_{\mathbf{i}}^{\prime}, \mathbf{x}_{\mathbf{0}}\right)}\left[T_{d}\left(\mathbf{x}_{\mathbf{i}}^{\prime}, \mathbf{x}_{\mathbf{0}}, t^{\prime}\right)\right]^{\mathrm{inv}} R\left(\mathbf{x}_{\mathbf{0}}, \mathbf{x}_{\mathbf{0}}^{\prime \prime}, t-t^{\prime}\right) d t^{\prime} \\
& -r \int_{\partial D_{0}} d \mathbf{x}_{\mathbf{0}} \int_{-t}^{-t_{d}^{\epsilon}\left(\mathbf{x}_{\mathbf{i}}^{\prime}, \mathbf{x}_{\mathbf{0}}\right)}\left[T_{d}\left(\mathbf{x}_{\mathbf{i}}^{\prime}, \mathbf{x}_{\mathbf{0}}, t^{\prime}\right)\right]^{\text {inv }} R\left(\mathbf{x}_{\mathbf{0}}, \mathbf{x}_{\mathbf{0}}^{\prime \prime}, t+t^{\prime}\right) d t^{\prime},
\end{aligned}
$$

for $t<t_{d}\left(\mathbf{x}_{\mathbf{i}}^{\prime}, \mathbf{x}_{\mathbf{0}}^{\prime \prime}\right)$ and $M_{k}\left(\mathbf{x}_{\mathbf{i}}^{\prime}, \mathbf{x}_{\mathbf{0}}^{\prime \prime},-t\right)=0$ for $t \geq t_{d}\left(\mathbf{x}_{\mathbf{i}}^{\prime}, \mathbf{x}_{\mathbf{0}}^{\prime \prime}\right)$. In contrast to the algorithm of Wapenaar et al. (2013a), we use $R$ instead of $R_{0}$, and we also include the reflection from the free surface (the last term in equation A-9). After convergence, we substitute the coda $M$ into equation A-7 to yield the focusing function $f_{2}$. The $f_{2}$ solution is then used in equation A-5 to obtain the two-way Green's function.

\section{REFERENCES}

Amundsen, L., 2001, Elimination of free-surface related multiples without need of the source wavelet: Geophysics, 66, 327-341, doi: 10.1190/1 .1444912 .

Bakulin, A., and R. Calvert, 2006, The virtual source method: Theory and case study: Geophysics, 71, no. 4, SI139-SI150, doi: 10.1190/1.2216190.

Behura, J., K. Wapenaar, and R. Snieder, 2012, Newton-Marchenko-Rose imaging: 82nd Annual International Meeting, SEG, Expanded Abstracts, doi: 10.1190/segam2012-1531.1.

Broggini, F., and R. Snieder, 2012, Connection of scattering principles: A visual and mathematical tour: European Journal of Physics, 33, 593-613, doi: 10.1088/0143-0807/33/3/593. 
Broggini, F., R. Snieder, and K. Wapenaar, 2012, Focusing the wavefield inside an unknown 1D medium: Beyond seismic interferometry: Geophysics, 77, no. 5, A25-A28, doi: 10.1190/geo2012-0060.1.

Broggini, F., R. Snieder, K. Wapenaar, and J. Behura, 2014, Wavefield autofocusing and imaging with multidimensional deconvolution: Numerical examples for reflection data with internal multiples: Geophysics, 79, no. 3, WA107-WA115, doi: 10.1190/geo2013-0307.1.

Claerbout, J., 1971, Toward a unified theory of reflector mapping: Geophysics, 36, 467-481, doi: 10.1190/1.1440185.

Claerbout, J., 1985, Imaging the earths interior: Blackwell Scientific Publications.

Jiang, Z., J. Sheng, J. Yu, G. Schuster, and B. Hornby, 2007, Migration methods for imaging different-order multiples: Geophysical Prospecting, 55, 1-19, doi: 10.1111/j.1365-2478.2006.00598.x.

Muijs, R., J. O. A. Robertsson, and K. Holliger, 2007a, Prestack depth migration of primary and surface-related multiple reflections. Part I: Imaging: Geophysics, 72, no. 2, S59-S69, doi: 10.1190/1.2422796.

Muijs, R., J. O. A. Robertsson, and K. Holliger, 2007b, Prestack depth migration of primary and surface-related multiple reflections. Part II: Identification and removal of residual multiples: Geophysics, 72, no. 2, S71S76, doi: 10.1190/1.2424544.

Rose, J., 2002a, Single-sided autofocusing of sound in layered materials: Inverse Problems, 18, 1923-1934, doi: 10.1088/0266-5611/18/6/329.

Rose, J., 2002b, Time reversal, focusing and exact inverse scattering, in $\mathrm{M}$ Fink, W. A.. Kuperman, J.-P. Montagner, and A. Tourin, eds., Imaging of complex media with acoustic and seismic waves: Springer, 97-106.

Schuster, G. T., Z. Jiang, and J. Yu, 2003, Imaging the most bounce out of multiples: 65th Annual International Meeting and Conference, EAGE, Extended Abstracts, P4.

Slob, E., K. Wapenaar, F. Broggini, and R. Snieder, 2014, Seismic reflector imaging using internal multiples with Marchenko-type equations: Geophysics, 79, no. 2, S63-S76, doi: 10.1190/geo2013-0095.1.

Van der Neut, J., J. Thorbecke, K. Mehta, E. Slob, and K. Wapenaar, 2011 Controlled-source interferometric redatuming by crosscorrelation and multidimensional deconvolution in elastic media: Geophysics, 76, no. 4, SA63-SA76, doi: 10.1190/1.3580633.
Verschuur, D., A. Berkhout, and C. Wapenaar, 1992, Adaptive surface-related multiple elimination: Geophysics, 57, 1166-1177, doi: 10.1190/1 1443330 .

Wapenaar, C. P. A., and J. L. T. Grimbergen, 1996, Reciprocity theorems for one-way wavefields: Geophysical Journal International, 127, 169-177, doi: 10.1111/j.1365-246X.1996.tb01542.x.

Wapenaar, K. F. Broggini, E. Slob, and R. Snieder, 2013a, Three-dimensional single-sided Marchenko inverse scattering, data-driven focusing, Green's function retrieval, and their mutual relations: Physical Review Letters, 110, 084301, doi: 10.1103/PhysRevLett.110.084301.

Wapenaar, K., E. Slob, J. van der Neut, J. Thorbecke, F. Broggini, and R. Snieder, 2013b, Three-dimensional Marchenko equation for Green's function retrieval beyond seismic interferometry: 83rd Annual International Meeting, SEG, Expanded Abstracts, 4573-4578.

Wapenaar, K., , F. Broggini, and R. Sniederet al., 2011, A proposal for model-independent $3 \mathrm{D}$ wave field reconstruction from reflection data: 81st Annual International Meeting, SEG, Expanded Abstracts, 37883792.

Wapenaar, K., and J. Fokkema, 2006, Green's function representations for seismic interferometry: Geophysics, 71, no. 4, SI33-SI46, doi: 10.1190/1 .2213955 .

Wapenaar, K., J. Fokkema, M. Dillen, and P. Scherpenhuijsen, 2000, Oneway acoustic reciprocity and its applications in multiple elimination and time-lapse seismics: 80th Annual International Meeting, SEG, Expanded Abstracts, 2377-2380.

Wapenaar, K., J. Thorbecke, and D. Draganov, 2004, Relations between reflection and transmission responses of three-dimensional inhomogeneous media: Geophysical Journal International, 156, 179-194, doi: 10.1111/j .1365-246X.2003.02152.x.

Wapenaar, K., J. Thorbecke, J. van der Neut, F. Broggini, E. Slob, and R Snieder, 2014, Green's function retrieval from reflection data, in absence of a receiver at the virtual source position: Journal of the Acoustical Society of America, 135, 2847-2861, doi: 10.1121/1.4869083.

Weglein, A., and W. Dragoset, 2007, Multiple attenuation: SEG 23, of Geophysics Reprint Series, 482 